\title{
Preface
}

\section{The Unfavorable Outcome: Here We Conquer}

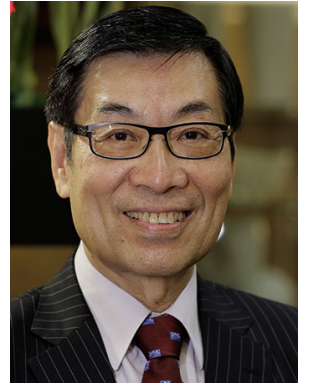

Fu-Chan Wei, MD, FACS

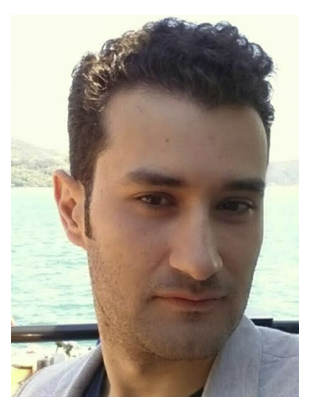

Nidal Farhan AL Deek, MSc, MD

Editors
Microsurgical reconstruction of the head and neck defects is the gold standard of treatment. Success rates are in the region of $98 \%$ in most of the major referral centers. Traditionally, success is defined by the patency of microvascular anastomosis and free flap viability. However, as we grow more critical about our success, its definition has evolved to refer to the successful restoration of function and appearance with improved patient quality of life.

Achieving a successful reconstruction beyond flap survival is, therefore, a challenging, experience-demanding endeavor that necessitates a comprehensive understanding of the unfavorable outcomes. This includes why and how they happen as well as strategies to avoid and manage them.

Information on the unfavorable outcomes after a microsurgical head and neck reconstruction gone wrong despite successful free flap surgery is lacking. The paucity of information in the literature is the impetus for this special issue of Clinics in Plastic Surgery. Our aim is to provide an immense, experience-based reflection with sincere accounts from the experts on the unfavorable outcomes after microsurgical reconstruction of the head and neck. And, our hope is that this work will help guide surgeons to achieve success in restoring human dignity with good function and aesthetics.
The content is organized into three subcategories, including (1) the unfavorable outcomes in microsurgical head and neck reconstruction: what they are and why they happen; (2) specific discussion on the unfavorable outcomes per subsite, empowered by case-based illustrations to educate on the untoward outcomes or demonstrate how international experts in world-renowned centers approach these problems; and (3) avoidance and management of specific unfavorable results commonly seen in microsurgical head and neck reconstruction.

We are very proud to say that each of the authors is an authority in head and neck microsurgical reconstruction. This issue is a fusion of knowledge gained from years of experience in a wealth of varying disciplines such as head and neck surgery, maxillofacial surgery, and plastic surgery.

We would specifically like to extend our sincerest gratitude to them for making this a highlight issue of the prestigious and renowned series Clinics in Plastic Surgery.

We also would like to thank the team from Elsevier for their great effort and kind assistance in making this work a reality. In particular, we would like to thank Jessica McCool and Don Mumford for their support and help; it was a great pleasure working with them.

We sincerely hope that you, the reader, will find this issue useful in planning the reconstruction in 
such a way that results in fewer complications and enhances your patient quality of life.

Fu-Chan Wei, MD, FACS Department of Plastic and Reconstructive Surgery Chang Gung Memorial Hospital Chang Gung Medical College Chang Gung University 199 Tun-Hwa North Road Taipei 10591, Taiwan
Nidal Farhan AL Deek, MSc, MD Department of Plastic and Reconstructive Surgery Chang Gung Memorial Hospital Chang Gung Medical College Chang Gung University 199 Tun-Hwa North Road Taipei 10591, Taiwan

E-mail addresses: fuchanwei@gmail.com (F.-C. Wei) nidaldeek@gmail.com (N.F. AL Deek) 